\title{
Meningioangiomatosis: A Disease With Many Radiological Faces
}

\author{
Fábio A. Nascimento, Tim-Rasmus Kiehl, Peter C. Tai, Taufik A. Valiante, Timo Krings
}

Keywords: Meningioangiomatosis, Magnetic Resonance Imaging, Computed Tomography, Angiography, Refractory Epilepsy

\section{Case Description}

We report a 25-year-old man who started having focal seizures with occasional secondary generalization at 15 years of age. The events were characterized by head deviation to the right side and right arm clonic movements or tonic extension in conjunction with impairment of consciousness. These paroxysms occasionally evolved into secondarily generalized tonic-clonic seizures. Initial investigation included computed tomography and magnetic resonance imaging (MRI) of the brain. The former showed left parietal clumped calcifications (Figure 1A), whereas the latter revealed a lesion with heterogeneous signal intensity in the left superior parietal lobe and significant contrast enhancement (Figure 1B-D). Further investigation with magnetic resonance angiography (MRA) and cerebral angiography was conducted. MRA demonstrated decreased arteriovenous transit time (Figure 1E) and angiography revealed a dysplastic terminal branch of the left middle cerebral artery as well as faint parenchymal blush in the left parietal lobe with no prominent draining vein (Figure 1F). Scalp electroencephalography showed a seizure generator with concordant topography. Based on all of these studies, a diagnosis of meningioangiomatosis was suspected. The patient subsequently developed refractory epilepsy and underwent an awake craniotomy with intraoperative electrocorticography-guided lesionectomy. Postoperative histological examination confirmed the diagnosis of meningioangiomatosis (MA) (Figure 2).

MA, a rare lesion of the leptomeninges and underlying cerebral cortex, was first described at the beginning of the 20th century by Bassoe et al. ${ }^{1}$ Originally, MA was linked with neurofibromatosis type 2; however, this condition can also occur sporadically. Patients with sporadic MA usually present with seizures, which are typically refractory to antiepileptic drug treatment. They may also have headaches at an early age (i.e. childhood or young adulthood). In contrast, patients with neurofibromatosis type 2-associated MA tend to remain asymptomatic throughout life. ${ }^{2-4}$ Histopathologically, the lesions contain a perivascular proliferation of meningothelial and fibroblast-like cells, entrapped glial islands, and focal microcalcifications. ${ }^{4}$ In terms of neuroimaging, MA is fairly diverse in its presentation. ${ }^{5}$ Nonetheless, according to a recent study that reviewed imaging features of 101 previously published MA patients, there are a few radiological characteristics that are suggestive of this condition. On brain computed tomography, these characteristics consist of low density, contrast enhancement, and presence of intralesional calcifications. On brain MRI, they comprise hypointensity on T1, hyperintensity on T2, and enhancement on T1. Regarding cerebral angiography, most patients appear to have a normal study. However, possible abnormalities include hypervascularity, abnormal vessels, and an avascular mass. ${ }^{5}$

MA is a difficult diagnosis to make, mainly because of nonspecific imaging findings. Therefore, we highlight the paramount importance of being familiar with the broad spectrum of possible radiological presentations, particularly in the most frequently used neuroimaging modalities (computed tomography, MRI/MRA, and cerebral angiography). Moreover, we intend to remind clinicians to include MA in the differential diagnoses of intracortical lesions and medically refractory seizures, especially in young adults.

\section{Disclosures}

The authors have nothing to disclose.

\section{Statement of Authorship}

FAN undertook study concept and design, data analysis and interpretation, and drafting of the manuscript. TRK undertook data analysis and interpretation, study supervision, revising the manuscript, and final approval. PCT undertook data analysis and interpretation, study supervision, revising the manuscript, and final approval. TV undertook data analysis and interpretation, study supervision, revising the manuscript, and final approval. TK undertook study concept and design, data analysis and interpretation, study supervision, revising the manuscript, and final approval. All authors accept responsibility for conduct of the research.

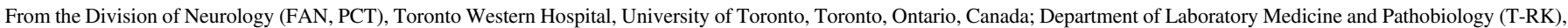

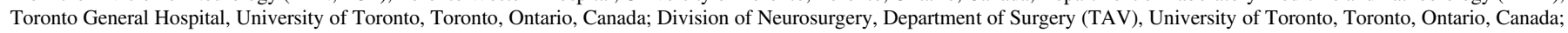

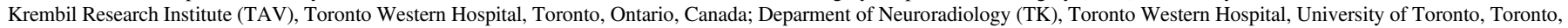
Ontario, Canada.

Received April 1, 2016. Date of Acceptance July 8, 2016.

Correspondence to: Fábio A. Nascimento, Toronto Western Hospital (TWH), 5W, 399, Bathurst Street, M5T 2S8, Toronto, Ontario, Canada.

E-mail: Nascimento.Fabio.A@gmail.com. 


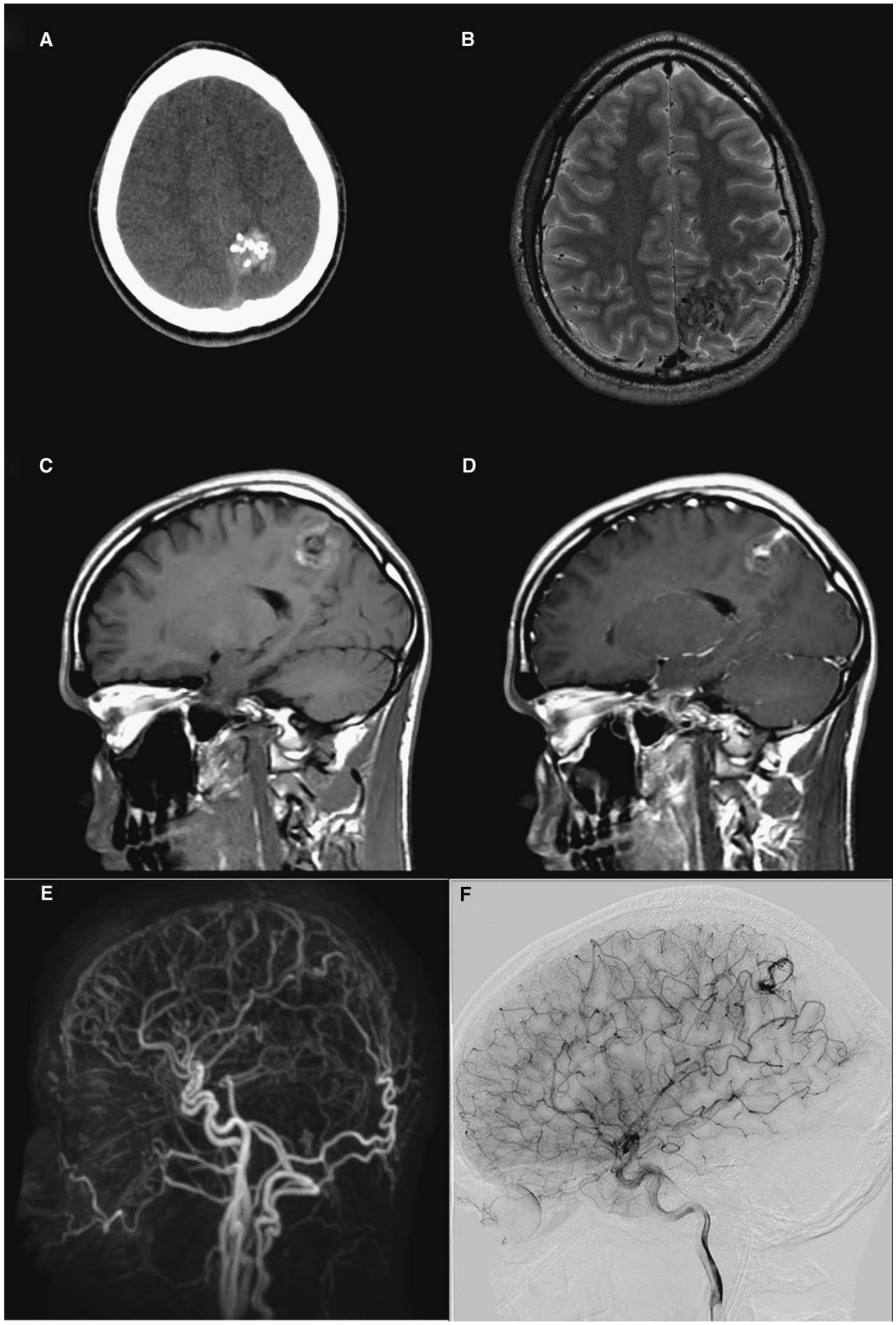

Figure 1: (A) Head computed tomography (CT) scan shows clumped calcifications at the left parietal topography. (B) Brain MRI: axial T2 reveals a heterogeneous, intra-axial, non-space-occupying lesion located within the left superior parietal lobe as well as signal heterogeneity. $(C, D)$ Contrast T1 imaging shows significant heterogeneous enhancement both in the lesion and in the adjacent leptomeningeal lining. (E) MRA demonstrates suspected early venous filling suggestive of decreased intralesional arteriovenous transit time. $(F)$ Angiography shows a dysplastic terminal part of the inferior parietal branch of the left middle cerebral artery and faint parenchymal blush in the left parietal lobe with no prominent draining vein, thus indicating that the vessel seen on MRA does not represent a vein but rather the dysplastic distal artery. 
A

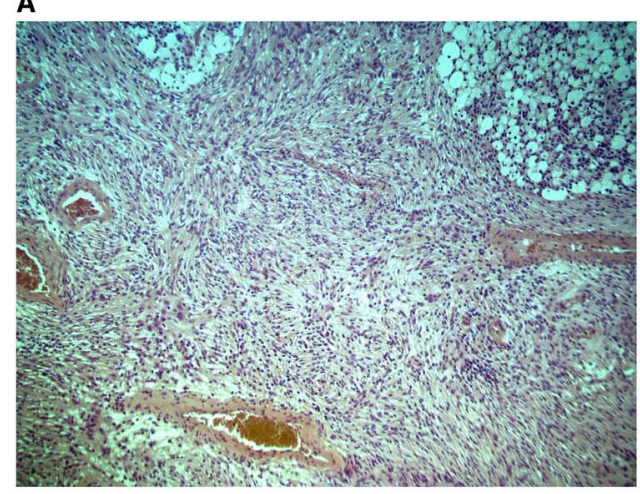

C

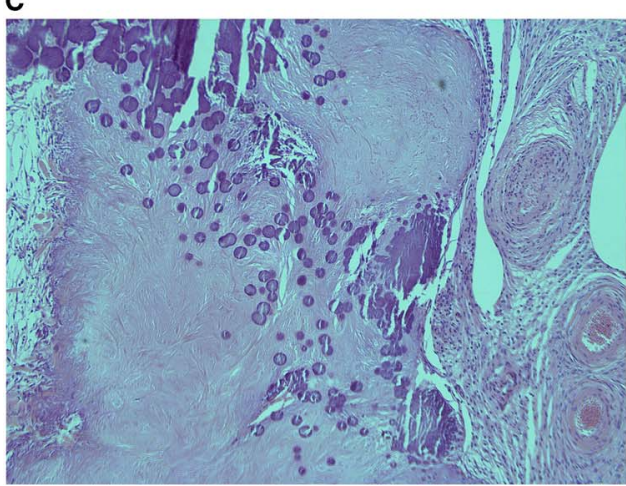

B

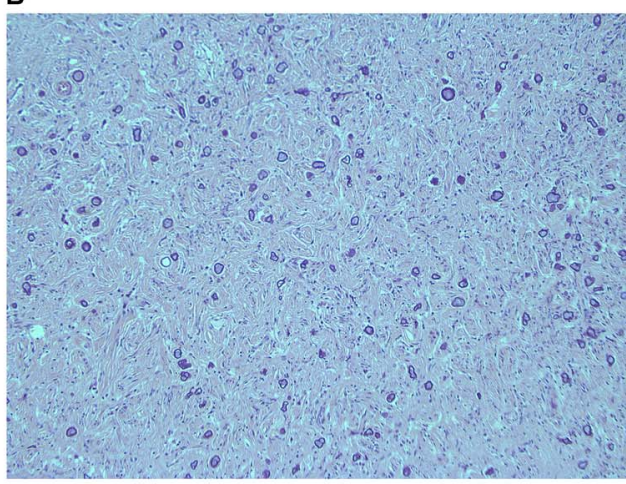

D

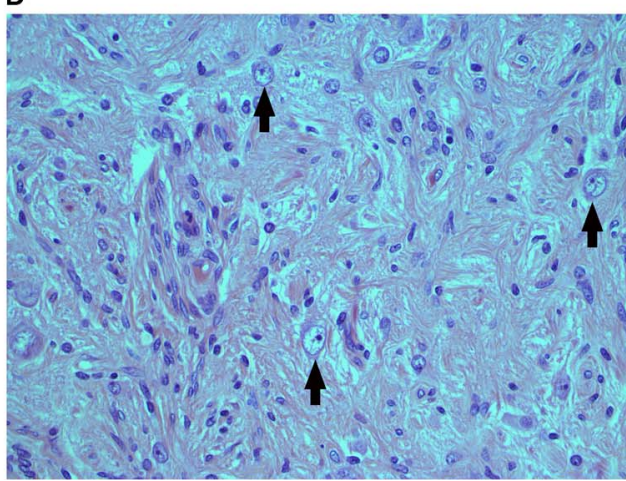

Figure 2: Variable histology of the lesion (all stains hematoxylin and eosin). (A) Low power (10x magnification) showing some areas with nearly pure proliferation of neoplastic meningothelial cells. Also present are collections of macrophages (upper right corner). (B) Infiltrated cortex with abundant psammomatous microcalcifications in part of the lesion $(10 \times) .(C)$ Large, densely sclerotic nodule that is beginning to calcify $(10 \times)$. (D) High power $(40 \times)$ demonstrating the neoplastic proliferation intermixed with residual brain cells such as cortical neurons (arrows).

\section{REFERENCES}

1. Bassoe P, Nuzum F. Report of a case of central and peripheral neurofibromatosis. J Nerv Ment Dis. 1915;42:785-96.

2. Wiebe S, Munoz DG, Smith S, Lee DH. Meningioangiomatosis. A comprehensive analysis of clinical and laboratory features. Brain. 1999;122:709-26.

3. Perry A, Kurtkaya-Yapicier O, Scheithauer BW, et al. Insights into meningioangiomatosis with and without meningioma: a clinicopathologic and genetic series of 24 cases. Brain Pathol. 2005;15:55-65.
4. Chen YY, Tiang XY, Li Z, Luo BN, Huang Q. Sporadic meningioangiomatosis-associated atypical meningioma mimicking parenchymal invasion of brain: a case report and review of the literature. Diagn Pathol. 2010;18:5-39.

5. Kashlan ON, Laborde DV, Davison L, et al. Meningioangiomatosis: a case report and literature review emphasizing diverse appearance on different imaging modalities. Case Rep Neurol Med. 2011;2011:361203. 